\title{
Nuclear and hadron matter equation of state within the induced surface tension approach
}

\author{
V.V. Sagun ${ }^{1, a}$, K.A. Bugaev ${ }^{1}$, A.I. Ivanytskyi ${ }^{1}$, D.R. Oliinychenko ${ }^{1,2}$, and I.N. Mishustin ${ }^{2,3}$ \\ ${ }^{1}$ Bogolyubov Institute for Theoretical Physics, Metrologichna str. 14 ${ }^{B}$, Kiev 03680, Ukraine \\ ${ }^{2}$ FIAS, Goethe-University, Ruth-Moufang Str. 1, 60438 Frankfurt upon Main, Germany \\ ${ }^{3}$ Kurchatov Institute, Russian Research Center, Akademika Kurchatova Sqr., Moscow, 123182, Russia
}

\begin{abstract}
We present a novel equation of state which is based on the virial expansion for the multicomponent mixtures with hard core repulsion. The suggested equation of state explicitly contains the surface tension which is induced by particle interaction. At high densities such a surface tension vanishes and in this way it switches the excluded volume treatment of hard core repulsion to its eigen volume treatment. The great advantage of the developed model is that the number of equations to be solved is two and it does not depend on the number of independent hard-core radii. Using the suggested equation of state we obtained a high quality fit of the hadron multiplicities measured at AGS, SPS, RHIC and ALICE energies and studied the properties of the nuclear matter phase diagram. It is shown the developed equation of state is softer than the gas of hard spheres and remains causal up to the several normal nuclear densities. Therefore, it could be applied to the neutron star interior modeling.
\end{abstract}

\section{Introduction}

Investigation of the strongly interacting matter equation of state $(\mathrm{EoS})$ is in focus of several physical communities. It is necessary to reliably model the process of nucleus-nucleus $(A+A)$ collisions at intermediate and high energies, and to study the properties of neutron star interiors. The hard-core repulsion is an important component of the hadronic and nuclear matter EoS because without it one cannot describe their properties at moderate and high baryonic densities. However, in the vast majority of models such a repulsion is treated using the Van der Waals approach which is inapplicable at the particle densities that are close to the transition region to quark gluon plasma (QGP). The problem is rooted in the wrong values of the third, the fourth and higher virial coefficients generated by the Van der Waals EoS. As it was shown in [1] a possible solution of this problem requires to account for the fact that at low densities an interparticle hard-core repulsion is well described by the excluded volume approximation, whereas the high density regime is controlled by the eigen volume of particles. The Van der Waals prescription is unable to switch between these two regimes and, therefore, it requires for an improvement. Another problem which is typical for all EoS with the hard-core repulsion is their non-causal behavior at high particle densities.

\footnotetext{
ae-mail: sagun@bitp.kiev.ua
} 
These facts motivated us to work out a novel EoS based on the analysis of the virial expansion for the multicomponent mixtures, i.e. for any number of hard-core radii of particle species. The main requirement for such an EoS was that it should be able to reproduce at least third and fourth virial coefficients of the gas of hard spheres in order to go beyond the Van der Waals approximation. Below we show that the latter requirement allows us to formulate a thermodynamically consistent EoS which obeys the causality condition up to seven or eight normal nuclear densities. It is necessary to mention that the developed EoS has a remarkable advantage which is important for practical applications: compared to other EoS with multicomponent hard-core repulsion the new one has a form of two coupled nonlinear equations for any, even infinite, number of particle species. The contribution of surface tension induced by the particle interaction is a principally new element of suggested approach.

In addition, using the novel EoS we would like to reinvestigate the question raised in [2] whether or not the deeper minimum of $\chi^{2} / d o f$ exist at very high temperature. Since, this question is very important for the whole heavy ion phenomenology, here we reanalyze the ALICE data on hadron multiplicities [3-9] with the novel model, which is able to go beyond the usual Van der Waals approximation.

The work is organized as follows. In the next section the theoretical basis of the present model is briefly outlined. In Sections 3 and 4 we present the application of the proposed model to the hadron and nuclear matter EoS, respectively. Section 5 is devoted to the conclusions.

\section{Model formulation}

The present model is formulated using a consistent treatment of the second virial coefficients for an ensemble of any, even infinite, number $N$ of hard-core radii either nuclear or hadron fragments of all sizes. Such an approach allows one to explicitly account for the many-body effects and to deduce that the hard-core interaction between the constituents generate an additional contribution into the (induced) surface tension free energy. Thermodynamically consistent EoS developed in [1] is a system of coupled equations between the pressure $p$ of considered system and the induced surface tension coefficient $\Sigma$ which has the form

$$
\begin{aligned}
p & =T \sum_{k=1}^{N} \phi_{k} \exp \left[\frac{\mu_{k}}{T}-V_{k} \frac{p}{T}-S_{k} \frac{\Sigma}{T}\right], \\
\Sigma & =T \sum_{k=1}^{N} R_{k} \phi_{k} \exp \left[\frac{\mu_{k}}{T}-V_{k} \frac{p}{T}-S_{k} \alpha \frac{\Sigma}{T}\right] .
\end{aligned}
$$

Here $\mu_{k}, m_{k}$ and $R_{k}$ are, respectively, the chemical potential, the mass and the hard-core radius of the $k$-sort of particles. Evidently, $V_{k}=\frac{4}{3} \pi R_{k}^{3}$ and $S_{k}=4 \pi R_{k}^{2}$ denote, respectively, the hard-core eigen volume and hard-core eigen surface of particle of sort $k$. The actual parameterization of the one-particle thermal density $\phi_{k}(T, m, g)$ corresponding to the particle of the mass $m_{k}$ and the degeneration factor $g_{k}$ depends on the nature of constituents and, hence, it is discussed below in details. The summations in Eqs. (1) and (2) are made over all sorts of particles and antiparticles which are considered as independent species.

The dimensionless parameter $\alpha$ is introduced in (2) due to the non-uniquness of the Van der Waals extrapolation to high densities [1]. This parameter accounts for the high density terms which modify the Van der Waals EoS to a more realistic one. As it was shown in [1] to reproduce the physically correct phase diagram properties of nuclear matter such a parameter should obey the inequality $\alpha>1$. The physical meaning of $\alpha$ is a switcher between the excluded volume and the eigen volume regimes. 
Indeed, using the following relation

$$
\Sigma=p R \exp \left[-4 \pi R^{2} \cdot(\alpha-1) \frac{\Sigma}{T}\right],
$$

between the total pressure $p$ and the induced surface tension coefficient $\Sigma$ for the one component mixture, i.e. in case when all particles have the same hard-core radius $R$, one obtains Eq. (3) from the system (1-2). With the help of (3) one can rewrite the system pressure for the one component case as

$$
p=T \sum_{k=1}^{N} \phi_{k} \exp \left[\frac{\mu_{k}}{T}-v^{e f f} \frac{p}{T}\right], \quad v^{e f f}=v\left[1+3 \cdot \exp \left(-3 v \cdot(\alpha-1) \frac{\Sigma}{T R}\right)\right],
$$

where we introduced an effective excluded volume of hadrons $v^{\text {eff }}$ which is defined by their eigen volume $v=\frac{4}{3} \pi R^{3}$. It is easy to see that in the low density limit $\mu_{k} \rightarrow-\infty$ and, hence, one finds $\frac{\Sigma v}{T R} \rightarrow 0$ and $v^{e f f} \simeq 4 v$, i.e. Eq. (4) for $v^{\text {eff }}$ correctly reproduces the excluded volume of the one component case. On the other hand, in the high density limit $\mu_{k} / T \gg 1$ and, hence, $\frac{\Sigma v}{T R} \gg 1$, i.e. for $\alpha>1$ the exponential function on the right hand side of Eq. (4) vanishes and the effective excluded volume becomes equal to the eigen volume, i.e. $v^{\text {eff }} \simeq v$.

The value of $\alpha$ was determined by comparing the system (1-2) with the induced surface tension (IST EoS hereafter) for the point-like pions and for baryons having the same hard-core radius $0.4 \mathrm{fm}$ with the famous Carnahan-Starling (CS) EoS [10]. As one can see from left panel on Fig. 1 up to the baryonic density $\rho \sim 0.65 \mathrm{fm}^{-3}$ the IST EoS with $\alpha=1.25$ reproduces the speed of sound $c_{S}$ of the CS EoS. From the right panel of Fig. 1 one can see that for temperature $150 \mathrm{MeV}$ the proposed EoS obeys causality up to baryonic density $\rho \sim 1.2 \mathrm{fm}^{-3}$, i.e up to seven and a half values of the normal nuclear density $\rho_{0} \simeq 0.16 \mathrm{fm}^{-3}$.
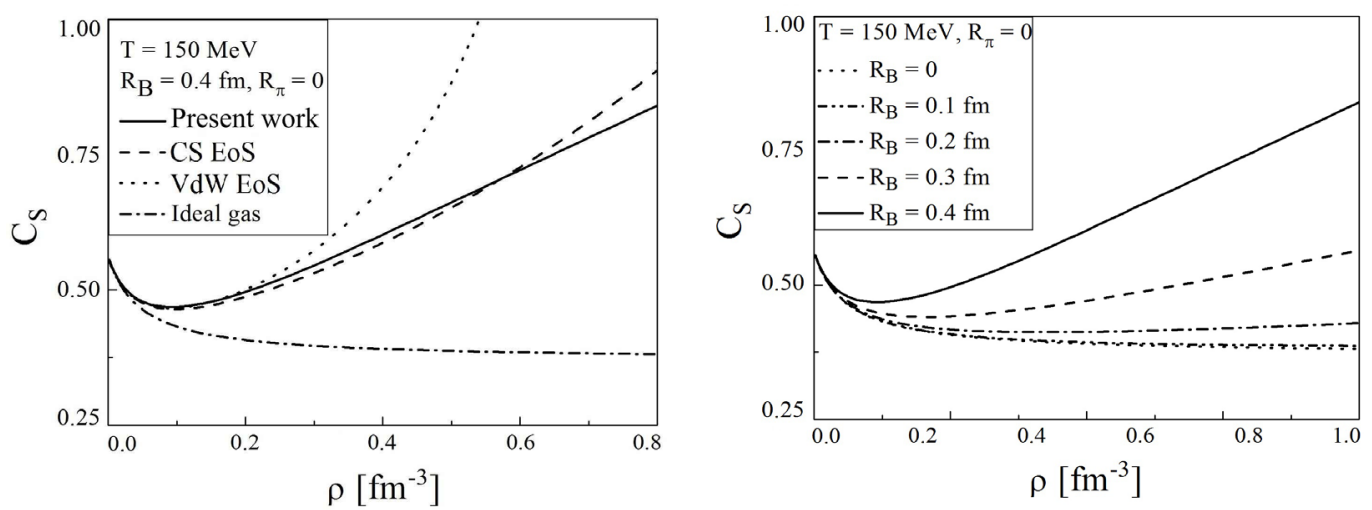

Figure 1. The speed of sound as a function of baryonic density for $\mathrm{T}=150 \mathrm{MeV}$ is shown for a mixture of point-like pions and baryons having the hard-core radius of $0.4 \mathrm{fm}$ for several EoS (left panel) and different radii of baryons (right panel) for IST EoS. In the left panel the ideal gas EoS (dash dotted curve), the Van der Waals EoS (dotted curve), the CS EoS (long dashed curve) and the IST EoS (solid curve) curves are shown.

After fixing the parameter $\alpha$ one can apply the IST EoS to study the properties of the hadron resonance gas and the ones of nuclear matter. The new EoS is essentially more effective compared to the traditional multicomponent Hadron Resonance Gas Model (MHRGM) [11-14] and traditional 
Statistical Multifragmentation Model (SMM) [15-18], since it can be easily used for any number of independent hard-core radii.

\section{HRGM with the induced surface tension}

Now we apply the IST EoS to the description of the hadron multiplicities measured in the central collisions of heavy ions. The total chemical potential of hadron sort $k$ is

$$
\mu_{k}=\mu_{B} B_{k}+\mu_{I 3} I_{3 k}+\mu_{S} S_{k}
$$

where $B_{k}, \mu_{B}, S_{k}, \mu_{S}, I_{3 k}, \mu_{I 3}$ are, respectively, the baryonic, the strange and the isospin third projection charges and chemical potentials. All these parameters describe the chemical freeze-out (CFO) curve on which all multiplicities form. The one-particle thermal density $\phi_{k}$ in Eqs. (1) and (2) accounts for the Breit-Wigner mass distribution and is written in the Boltzmann approximation

$$
\phi_{k}=g_{k} \gamma_{S}^{\left|s_{k}\right|} \int_{M_{k}^{T h}}^{\infty} \frac{d m}{N_{k}\left(M_{k}^{T h}\right)} \frac{1}{\left(m-m_{k}\right)^{2}+\Gamma_{k}^{2} / 4} \int \frac{d^{3} p}{(2 \pi)^{3}} \exp \left[-\frac{\sqrt{p^{2}+m^{2}}}{T}\right] .
$$

Here $g_{k}$ and $m_{k}$ are, respectively, the degeneracy factor and the mass of the $k$-sort of hadrons, $\gamma_{S}$ is the strangeness suppression factor [19], $\left|s_{k}\right|$ is the number of valence strange quarks and antiquarks in this kind of hadrons, $N_{k}\left(M_{k}^{T h}\right) \equiv \int_{M_{k}^{T h}}^{\infty} \frac{d m}{\left(m-m_{k}\right)^{2}+\Gamma_{k}^{2} / 4}$ denotes a corresponding normalization, while $M_{k}^{T h}$ the decay threshold mass of the hadrons of $k$-sort.

Experimentally detected hadron multiplicity of each hadron is the sum of a thermal and the decay contributions, i.e. $n_{X}^{\text {tot }}=n_{X}^{\text {th }}+n^{\text {decay }}=n_{X}^{\text {th }}+\sum_{Y} n_{Y}^{\text {th }} \operatorname{Br}(Y \rightarrow X)$, where $\operatorname{Br}(Y \rightarrow X)$ is the decay branching ratio of the $Y$-th hadron into the hadron $X$ (for more details see [14]). In addition, it is supposed for convenience that $B R(X \rightarrow X)=1$. All the parameters used in the fitting of data (the masses $m_{i}$, the widths $\Gamma_{i}$, the degeneration factors $g_{i}$ and the probabilities of decays for all strong decay channels) were taken from the particle tables of the thermodynamic code THERMUS [20].

The best fit criterion is a minimum of $\chi^{2}=\sum_{k} \frac{\left(r_{k}^{\text {theor }}-r_{k}^{e x p}\right)^{2}}{\sigma_{k}^{2}}$, where $r_{k}^{\text {exp }}$ is an experimental value of $\mathrm{k}$-th particle ratio, $r_{k}^{\text {theor }}$ is our prediction and $\sigma_{k}$ is a total error of experimental value. Using the value $\alpha=1.25$ we employed the IST EoS to fit the 111 independent hadronic multiplicity ratios measured in the central nuclear collisions for the center of mass collision energies $\sqrt{s_{N N}}=2.7,3.3,3.8,4.3,4.9$, 6.3 7.6, 8.8, 9.2, 12, 17, 62.4, 130 and $200 \mathrm{GeV}$ were taken from Ref. [14]. For the AGS, SPS, RHIC data the $\gamma_{S}$ parameter was included in the fit.

The lowest $\chi^{2} /$ dof value corresponds to following hard core radii of baryons $R_{b}=0.365 \mathrm{fm}$, mesons $R_{m}=0.42 \mathrm{fm}$, pions $R_{\pi}=0.15 \mathrm{fm}$, kaons $R_{K}=0.395 \mathrm{fm}$ and $\Lambda$-hyperons $R_{\Lambda}=0.085 \mathrm{fm}$ within $\chi^{2} /$ dof $=57.099 / 55 \simeq 1.038$ which is about $9 \%$ larger than the $\chi^{2} /$ dof found earlier in [14] for the MHRGM. Compared to the values found by the MHRGM, one sees that only pionic hard-core radius increased by $50 \%$, while the hard-core radius of $\Lambda$ hyperons diminished by $20 \%$. The most important thing is that these radii remain essentially smaller compared to $R_{b}, R_{m}$ and $R_{K}$. The latter hard-core radii are practically unchanged.

Exactly the same set of hard-core radii was used to the description of ALICE data. The IST EoS was applied to the description of the hadron multiplicities at midrapidity $\left.\frac{d N}{d y}\right|_{|y|<0.5}$ measured by the ALICE detector at $\sqrt{s_{N N}}=2.76 \mathrm{TeV}$ in $\mathrm{Pb}+\mathrm{Pb}$ collisions. The experimental data were taken from Refs. $[3-9,21]$. All hadron multiplicities were $p_{T}$-integrated and those ones at low $p_{T}$ were 


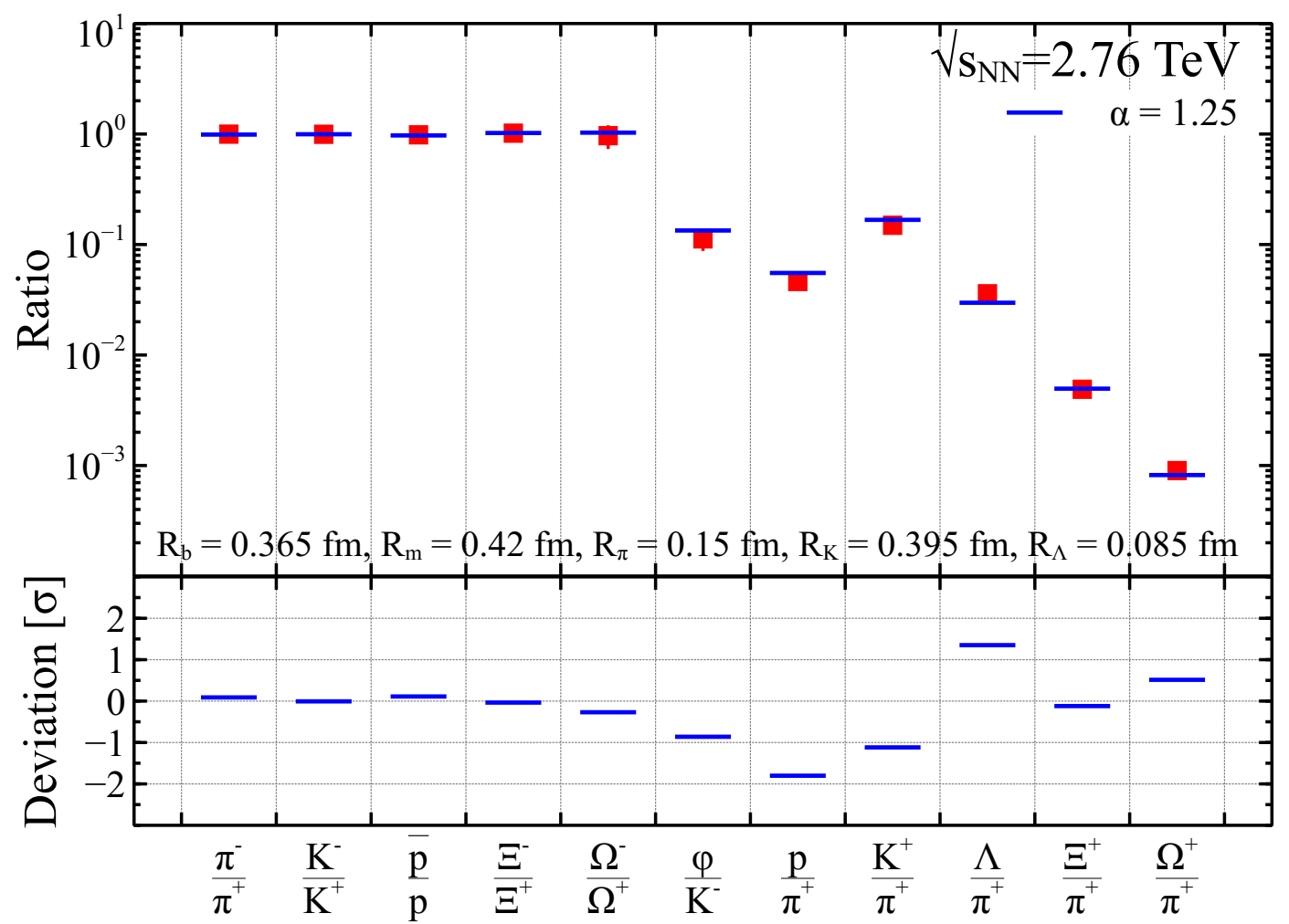

Figure 2. ALICE data fit by the IST EOS with the new set of hard-core radii. The found CFO temperature is $T_{C F O} \simeq 154 \pm 7 \mathrm{MeV}$. The (anti)nuclei rations are not included in the fit and its quality is $\chi^{2} /$ dof $\simeq 7.7 / 5 \simeq 1.54$. The upper panel shows the fit of the ratios, while the lower panel shows the deviation between data and theory in the units of estimated error.

extrapolated by the blast wave fit in each centrality bin while extrapolation error was included in the systematic errors.

The ratios of hadron multiplicities $r=\frac{A}{B}$ were constructed from species $A+\Delta A$ and $B+\Delta B$, while the estimation for a relative error $\epsilon_{r} \equiv \frac{\Delta r}{r}$ as $\sqrt{\epsilon_{A}^{2}+\epsilon_{B}^{2}}$ [22], where $\epsilon_{A}=\frac{\Delta A}{A}$ and $\epsilon_{B}=\frac{\Delta B}{B}$. It is, certainly, a slight overestimation, because a part of systematic errors, such as errors related to detector acceptance, usually is cancelled in the experimentally measured ratios. If the statistical and systematic errors $\Delta A_{\text {stat }}$ and $\Delta A_{\text {sys }}$ are given, we add them as $\Delta A=\sqrt{\Delta A_{\text {stat }}^{2}+\Delta A_{\text {sys }}^{2}}$ [22].

For the ALICE data we fixed $\gamma_{S}=1$, while all chemical potentials are set to zero. The best global fit of all hadronic multiplicity ratios is found for the CFO temperature $T_{C F O} \simeq 154 \pm 7 \mathrm{MeV}$ with $\chi^{2} / d o f \simeq 7.7 / 5 \simeq 1.54$ (see Fig. 2).

Using the IST EOS we found no traces of the high temperature minimum in comparison to Vovchenko-Stoecker results [2]. Our analysis shows that the high temperature minimum of $\chi^{2} /$ dof found in [2] is a consequence of extrapolating the Van der Waals EOS far beyond the bounds of its applicability. 


\section{SMM with the induced surface tension}

Originally, the IST EoS was first applied to the description of the nuclear matter properties on the basis of an exactly solvable version of the SMM $[17,18]$. Such a model is dealing with the nucleons with the mass $m \simeq 940 \mathrm{MeV}$ and the eigen volume $V_{1}=\rho_{0}^{-1}$ (where $\rho_{0}$ denotes the normal nuclear density at $T=0$ and zero pressure) and the composite nuclear fragments of any number of nucleons $k \geq 2$. Their eigen volume is $V_{k}=k V_{1}$ and the corresponding surface area is $S_{k} \sim k^{\frac{2}{3}}$.

To connect the above system of equations (1-2) for pressure and induced surface tension coefficient with the gaseous phase pressure of the SMM, we used the parameterization of the one-particle thermal densities of all $k$-nucleon fragments defined as

$$
\begin{aligned}
\phi_{1} & =z_{1}\left[\frac{m T}{2 \pi}\right]^{\frac{3}{2}} \exp \left[-\frac{\sigma(T)}{T}\right] \\
\phi_{k \geq 2} & =g\left[\frac{m T}{2 \pi}\right]^{\frac{3}{2}} \frac{1}{k^{\tau}} \exp \left[\frac{\left(k p_{L} V_{1}-\mu_{k}\right)}{T}-\frac{\sigma(T)}{T} k^{\frac{2}{3}}\right] .
\end{aligned}
$$

Here $z_{1}=4$ is the degeneracy factor of nucleons, while the degeneracy factor for other fragments $g$ is, for simplicity, chosen to be 1 (see a discussion in [16]). In (8) $\mu_{k}=k \mu$ is the baryonic chemical potential of $k$-nucleon fragment, $\tau \simeq 1.9$ is the Fisher topological exponent and $\sigma(T)$ is the $T$-dependent eigen surface tension coefficient with the following parameterization

$$
\sigma(T)=\sigma_{0}\left[\frac{T_{c e p}-T}{T_{c e p}}\right] \operatorname{sign}\left(T_{c e p}-T\right),
$$

with critical temperature $T_{\text {cep }}=18 \mathrm{MeV}$ and eigen surface tension at zero temperature $\sigma_{0}=18 \mathrm{MeV}$. In contrast to the Fisher droplet model [23] and the usual SMM [15], in the IST SMM the value of the eigen surface tension (9) is negative above the critical temperature $T_{\text {cep }}$. An extended discussion on the validity of such a parameterization can be found in [24]. In order to consider compressible nuclear liquid the following parameterization of its pressure

$$
p_{L}=\frac{W(T)+\mu+a_{2}\left(\mu-\mu_{0}\right)^{2}+a_{4}\left(\mu-\mu_{0}\right)^{4}}{V_{1}},
$$

was suggested in [24]. Here $W(T)=W_{0}+\frac{T^{2}}{W_{0}}$ denotes the usual temperature dependent binding energy per nucleon with $W_{0}=16 \mathrm{MeV}$ [17] and the constants $\mu_{0}=-W_{0}, a_{2} \simeq 1.233 \cdot 10^{-2} \mathrm{MeV}^{-1}$ and $a_{4} \simeq 4.099 \cdot 10^{-7} \mathrm{MeV}^{-3}$. These constants are fixed in order to reproduce the properties of normal nuclear matter, i.e. at vanishing temperature $T=0$ and normal nuclear density $\rho=\rho_{0}$ the liquid pressure must be zero. It is worth to note that such a parametrization of the nuclear liquid pressure describes a compressible nuclear liquid and, in contrast to the original SMM formulation [15], it leads to a nonzero isothermal compressibility $\left.K_{T} \equiv \frac{1}{\rho} \frac{d \rho}{d p}\right|_{T}$.

The IST SMM was solved analytically and the first order phase transition of the liquid-gas type was found in [24]. It was proven that such a model has a tricritical endpoint with the temperature $T=18 \mathrm{MeV}$ and the baryonic density $\rho_{c e p}=\rho_{0} / 3$. The resulting phase diagram of the IST SMM for two sets of variables is shown in Fig. 3. As one can see from this figure the developed model with the surface tension induced by the repulsive interaction between the nuclear fragments in combination with a finite incompressibility of liquid phase has rather rich phase structure of the nuclear matter phase diagram. 

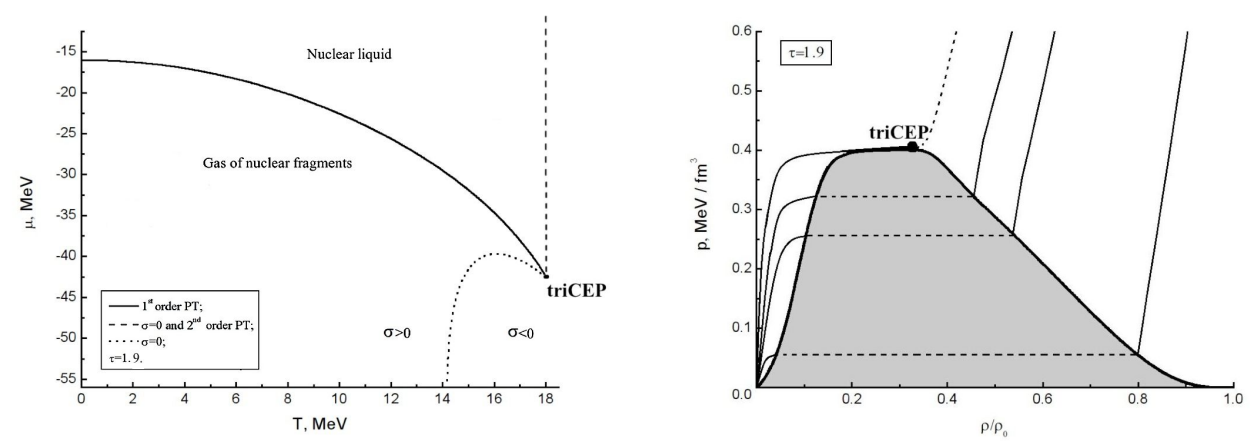

Figure 3. Phase diagram in $T-\mu$ plane (left panel) and $\rho-p$ plane (right panel) for $v=2$ and $\tau=1$.9. The 1 -st order PT corresponds to a solid curve (left panel) and grey area of a mixed phase (right panel). The long dashed line on the left panel shows a 2-nd order PT, while the short dashed curve indicates the nil line of the surface tension coefficient. The isotherms on the right panel are shown for $\mathrm{T}=11,16,17,18 \mathrm{MeV}$ from bottom to top. While at the critical temperature $T_{c e p}=18 \mathrm{MeV}$ and density $\rho / \rho_{0}=1 / 3$ there is a triCEP.

\section{Conclusions}

In this work we present a thermodynamically consistent approach which is able to account for the effects of hard-core repulsion in the ensemble of constituents (clusters) of different sizes. It is necessary to stress that the developed EoS allows one to go far beyond the usual Van der Waals approximation. We showed that the hard-core repulsion between the clusters unavoidably leads to an additional equation for the induced surface tension coefficient. Due to the non-uniqueness of the Van der Waals extrapolation to high particle densities a novel parameter $\alpha$ was introduced. We found that this parameter is the "switcher" between the excluded and eigen volume regimes. A detailed comparison with the famous CS EoS clearly demonstrates the validity of the IST EoS for the baryonic densities up to $0.65 \mathrm{fm}^{-3}$ and, while at higher densities it is softer than the CS EoS. In contrast to the conventional MHRGM the present one is mathematically much simpler because it consists only of two coupled equations independently of the number of different hard-core radii.

In order to demonstrate these advantages in practical applications, the IST EoS was used to study the properties of hadronic and nuclear matter. Using the developed approach we performed fit of the ALICE data measured at the center of mass collision energy $\sqrt{s_{N N}}=2.76 \mathrm{TeV}$ with overall fit quality $\chi^{2} / d o f \simeq 1.54$. The found value of the CFO temperature is $T_{C F O} \simeq 154 \pm 6 \mathrm{MeV}$. In comparison to the [2] we found no traces of the high temperature minimum for the ALICE data. The quality of the AGS, SPS and RHIC data description achieved in the present work is $\chi^{2} / d o f \simeq 64.8 / 60 \simeq 1.038$.

The IST EoS allowed us to formulate a more realistic version of the SMM in which for the first time the nuclear liquid is compressible. This property enabled us to generates the tricritical endpoint at the one third of the normal nuclear density which is in line with the liquid-gas phase transition in the ordinary liquids. It is necessary to stress that in such SMM the non-monotonic isotherms in the mixed phase region which are typical for the mean-field models are simply absent. Due to these new features the present model is more realistic than the standard SMM.

The discussed features of the proposed EoS make it applicable to the description of properties of hadronic, nuclear and neutron matter. 


\subsection{Acknowledgments}

V.V.S., K.A.B. and A.I.I. are thankful for the partial support of the program "On perspective fundamental research in high-energy and nuclear physics" launched by the Section of Nuclear Physics of National Academy of Sciences of Ukraine.

\section{References}

[1] V. V. Sagun, A. I. Ivanytskyi, K. A. Bugaev and I. N. Mishustin, Nucl. Phys. A 924, 24 (2014).

[2] V. Vovchenko and H. Stöcker, arXive:1512.08046v2 [hep-ph].

[3] B. Abelev et al. [ALICE Collaboration], Phys. Rev. C 88, 044910 (2013).

[4] B. B. Abelev et al. [ALICE Collaboration], Phys. Lett. B 728, 216 (2014); Erratum: [Phys. Lett. B 734, 409 (2014)]

[5] B. B. Abelev et al. [ALICE Collaboration], Phys. Rev. Lett. 111, 222301 (2013).

[6] A. G. Knospe [ALICE Collaboration], J. Phys. Conf. Ser. 509, 012087 (2014).

[7] J. Adam et al. [ALICE Collaboration], Phys. Rev. C 93, 024917 (2016).

[8] B. Dönigus [ALICE Collaboration], EPJ Web Conf. 97, 00013 (2015).

[9] J. Adam et al. [ALICE Collaboration], Phys. Lett. B 754, 360 (2016).

[10] N. F. Carnahan and K. E. Starling, J. Chem. Phys. 51, 635 (1969).

[11] A. Andronic, P. Braun-Munzinger and J. Stachel, Nucl. Phys. A 772, 167 (2006).

[12] K. A. Bugaev, D. R. Oliinychenko, A. S. Sorin and G. M. Zinovjev, Eur. Phys. J. A 49, 30 (2013).

[13] K. A. Bugaev et al., Europhys. Lett. 104, 22002 (2013).

[14] V. V. Sagun, Ukr. J. Phys. 59, 755 (2014).

[15] J. P. Bondorf et al., Phys. Rep. 257, 131 (1995) and references therein.

[16] K. A. Bugaev, Phys. Part. Nucl. 38 447, (2007).

[17] K. A. Bugaev, M. I. Gorenstein, I. N. Mishustin and W. Greiner, Phys. Rev. C 62, 044320 (2000).

[18] P. T. Reuter and K. A. Bugaev, Phys. Lett. B 517, 233 (2001).

[19] J. Rafelski, Phys. Lett. B 62, 333 (1991).

[20] S. Wheaton, J. Cleymans and M. Hauer, Comput. Phys. Commun. 180, 84 (2009).

[21] J. Stachel, A. Andronic, P. Braun-Munzinger and K. Redlich, J. Phys. Conf. Ser. 509, 012019 (2014).

[22] J. R. Taylor, “An introduction to error analysis”, University Science Book Mill Valley, California (1982).

[23] M. E. Fisher, Physics 3, 255 (1967).

[24] K. A. Bugaev, A. I. Ivanytskyi, V. V. Sagun and D. R. Oliinychenko, Phys. Part. Nucl. Lett. 10, 832 (2013). 